\title{
Interconnect for commodity FPGA clusters: standardized or customized?
}

\author{
A. Theodore Markettos, Paul J. Fox, Simon W. Moore, Andrew W. Moore \\ Computer Laboratory, University of Cambridge, UK \\ \{firstname.lastname\} @ cl.cam.ac.uk
}

\begin{abstract}
We demonstrate that a small library of customizable interconnect components permits low-area, high-performance, reliable communication tuned to an application, by analogy with the way designers customize their compute. Whilst soft cores for standard protocols (Ethernet, RapidIO, Infiniband, Interlaken) are a boon for FPGA-to-other-system interconnect, we argue that they are inefficient and unnecessary for FPGA-to-FPGA interconnect. Using the example of BlueLink, our lightweight pluggable interconnect library, we describe how to construct reliable FPGA clusters from hundreds of lower-cost commodity FPGA boards. Utilizing the increasing number of serial links on FPGAs demands efficient use of soft-logic, making domainoptimized custom interconnect attractive for some time to come.
\end{abstract}

\section{INTRODUCTION}

FPGA systems are hard to scale. A designer can use the largest FPGA money can buy, but this comes with a significant price penalty, as shown in Figure 1. Furthermore, it may only allow enlargement by a factor of two or four. A large workload may outgrow the FPGA by a factor of a hundred or more.

Additionally enlarging the FPGA does not necessarily increase resources. If a workload is memory-bound, the number of external memory interfaces may remain constant as an FPGA gets larger. Packaging limits constrain the number of I/O pins, so more DIMMs cannot be attached. Sooner or later a designer is forced to move to a multi-FPGA system.

In this paper we describe an approach to building FPGA clusters at scale, using commodity parts to minimize costs and engineering, and high-bandwidth serial transceivers for interconnect. We then consider interconnect protocols.

It would be natural to start by using a standard protocol such as Ethernet and standard soft cores. We assess whether such a standardized interconnect makes sense, or whether it is worth building a customized interconnect tailored to application requirements.

We illustrate this question with BlueLink, a custom FPGA interconnect toolkit that we designed for a specific application. We compare this with standard soft intellectual property (IP) cores to evaluate the merits of a custom approach.

Furthermore we explore how custom interconnect can make best use of commodity FPGA platforms and continue to scale in the future.

\section{BUILding AN FPGA Cluster}

When building a multi-FPGA system, the obvious approach is to put multiple FPGAs on the same printed circuit board (PCB). After all, FPGAs have hundreds of general-purpose I/O (GPIO) pins which can be used to connect them.

However there are a number of pitfalls to a multi-FPGA PCB. Firstly, designing such a board is a complex task. FPGAs have upward of 1000 pins to route, many high speed. For example, the Altera Stratix V PCIe Development board has 16 layers [3], which makes it costly to design and fabricate. FPGA power design is also complex - this single-FPGA board has 21 power rails with the highest current being 28 amps. This requires complex design and simulation - for professional designers a board takes about one man-year of design effort. FPGAs are typically found in advanced ball grid array packages, which also makes manufacturing difficult. In addition there is the headache of managing the whole process of parts procurement, production, test and debug.

Secondly, many such boards (especially commercial products) are not regular - each FPGA is not connected to the same peripherals. This requires a separate synthesis run for each FPGA in a cluster, which makes it difficult to scale to large numbers of FPGAs. Testing such boards is difficult, a fault may cause the whole board to fail, and repair is complex.

For example, Mencer et al. [11] used 64 Spartan 3 FPGAs on a large 8 -layer PCB $(320 \times 480 \mathrm{~mm})$. With 64-bit connections at $100 \mathrm{MHz}$ between FPGAs they achieved $6.4 \mathrm{Gbps}$ inter-FPGA bandwidth. They had to employ fault tolerance because replacing a faulty device is difficult. Furthermore, the engineering required for such boards makes them niche commercial products with high price tags. The DINI Group quote 'below $0.1 \varnothing$ per [ASIC] gate' for a ' 130 million ASIC gate' system containing 20 Stratix IV 530 devices - which makes the board cost around US\$130,000 [5].

Meanwhile, FPGA evaluation boards have become a commodity. These are commonly sold to engineers to prototype designs before final products, but they are increasingly being used as standalone platforms for research and development. Non-recurrent expenditure (NRE) from design and tool costs is amortized across the thousands of boards being shipped, reducing unit cost. If a board fails, it can simply be swapped out for another costing a few hundred or thousand dollars. It therefore makes economic sense to build a cluster with many commodity cards with some kind of interconnect. If this allows use of smaller FPGA parts, that ship in greater numbers and have better yield, it will further reduce cost. For example, in Figure 1 Cyclone $\mathrm{V}$ parts are roughly one sixth of the price of the comparable Stratix V.

\section{A. Application Partitioning}

When building a cluster, we must consider the applications that will run on it before we design its interconnect.

Some applications do not require any communication between FPGAs. These can be described as loosely coupled. 


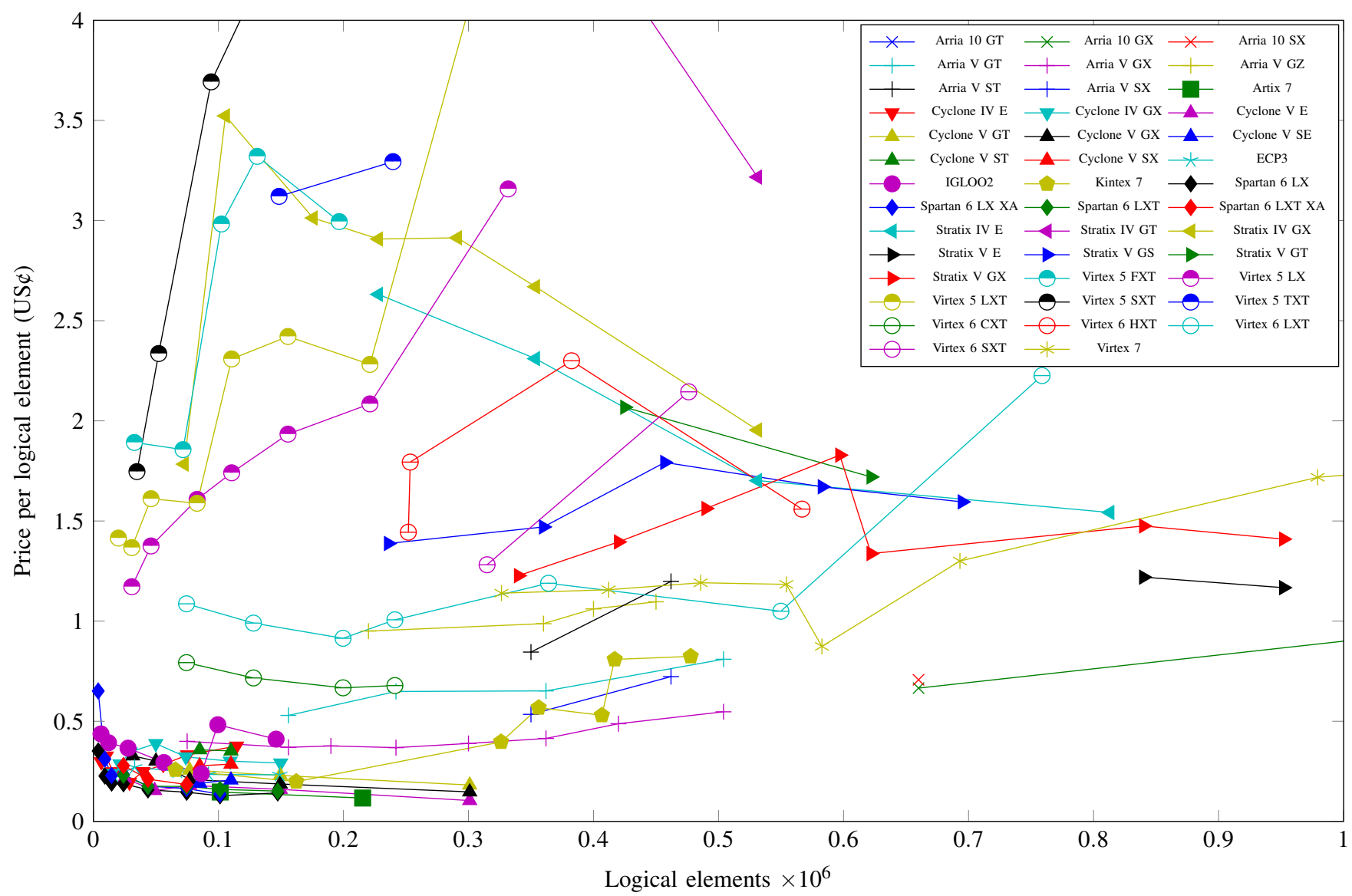

Fig. 1. FPGA pricing trends. Devices cluster in two board categories: smaller budget ranges with a lower cost per logic element, and premium parts which are considerably more expensive for the same number of elements. Within the budget range larger parts are cheaper per element. In some families in the premium range larger devices are disproportionately more expensive. Economics suggests that a cluster should use budget ranges wherever possible or alternatively smaller premium devices. Data: digikey.com [4]; we plot the median price for a given model and size, combining options of package and speed grade into a single point.

MapReduce fits this model, while FPGA examples include Bitcoin mining or OpenCL-based accelerators. The FPGA needs only a connection to a host PC, Scale is achieved by buying more PCs with FPGAs. Such a cluster is easy to build.

Other applications are tightly coupled. One example is gate-level system-on-chip simulation. This is very latencydependent: nodes operating in lock-step require single-cycle interconnect latency. This makes partitioning a hard problem, compounded by many possible fine-grained partitions.

To allow scale, it is better to use a coarser-grained architecture. If the number of possible partition combinations is reduced, partitioning becomes simpler. A higher level of abstraction may also permit relaxation of latency requirements, though latency can still be a major bottleneck. Lower area efficiency is mitigated as it is easier to add more hardware.

Additionally, a tiled approach can reduce FPGA synthesis time. If the FPGA bitfile is the same for each node, it only needs to be synthesized once. Different behaviour for each node can then be configured with different software, datasets and runtime configuration.

\section{B. Physical Interconnect}

We wish to build a cluster at scale, using hundreds of FPGAs on multiple boards. If connections between FPGAs are required, how should such a cluster be interconnected?
The simplest approach would be to use GPIO pins. These can be driven either single-ended or with low-voltage differential signalling (LVDS). However, the frequency they can be driven at is limited, about $1 \mathrm{GHz}$ in LVDS mode. Long parallel links are affected by signal integrity (constrains cable geometry for a good quality signal) and skew (signals arrive at different times). This means such cables are typically short (centimetres) and must employ careful (expensive) construction. This limits the size of cluster that can be built. Kono et al. [8] achieved a data rate of $4 \mathrm{Gbps}$ per link using HSMC connectors on Terasic DE4 boards and expensive proprietary ribbon cabling. With only two ports per board their cluster was forced to use a ring topology.

FPGAs now incorporate increasing numbers of high-speed serial transceivers. A device can have up to 96 transceivers each capable of up to $56 \mathrm{Gbps}$ (though $14 \mathrm{Gbps}$ is a more realistic maximum for lower cost parts). Many commodity I/O standards have shifted from parallel to serial interconnect (such as USB, SAS, SATA, PCI Express, etc). This means there are now cheap passive multi-gigabit serial cables on the market. Active repeater and optical cables are also available for longer distances. Such cables can be used as physical-layer bit-pipes, without using the intended protocol along them. All that is required is point-to-point cabling between whatever connectors the board manufacturer provided. 
Therefore we suggest that a cluster can be built at scale with the following properties:

- Commodity FPGA boards, to reduce cost and development time.

- Serial interconnect using FPGA transceivers.

- Low-cost commodity passive copper cabling between boards. If necessary, optical cabling for longer distances.

- Multi-hop routing, so that a fully-connected network is not required.

\section{Custom Communication?}

The question that remains is: what protocol should be used on the interconnect? Should you follow a standard, or is it worth designing your own? An FPGA designer may be comfortable with the idea of custom compute, where their compute is optimized for the workload. This is usually more effective than simply using a standard CPU soft-core on their FPGA. A natural extension of this would be custom communication, where communication is similarly optimized. Is it worth optimizing your communication, or is a standard core sufficient?

We shall consider a number of application examples, and the interconnect protocol that we designed for them. We will then compare our protocol with existing standards to identify the merits and pitfalls of each approach.

\section{Application CAse Studies}

The compute and communication requirements of an FPGA cluster may be different from other clusters such as datacenters or PC-based scientific compute. The following examples describe two applications that are suited to FPGA clusters and their communication requirements.

\section{A. Memory interconnect}

Consider a massive multiprocessor system using shared memory. A number of CPU cores (such as NIOS-II/Microblaze or custom processors) are located on each FPGA. Each FPGA board has up to $16 \mathrm{~GB}$ of DRAM. When a CPU core needs to access memory on another board, it must request a cache line from the other board. Each cache line might be 256 bits, which is set by the width of the interface to the memory controller. Thus a memory read consists of sending a 64-bit address and receiving a 256-bit response, or writing a 64-bit address and 256-bit value. Superscalar CPU architecture can mask a limited amount of memory latency, up to a few tens of cycles. A lost or further delayed memory transaction will cause a CPU to give an incorrect result or stall.

\section{B. Neural computing}

The human brain has approximately $10^{11}$ neurons with $10^{14}$ synaptic connections. Each neuron fires at about $10 \mathrm{~Hz}$. In some neuron models, neuron updates can be represented by a simple differential equation, but there are approximately $10^{15}$ synaptic messages per second. To achieve real-time operation the network must compute the state of every neuron, accounting for its $10^{3}$ incoming messages, every millisecond.
The need for timely delivery of large numbers of small, low-latency messages rules out classical CPUs, which do not have enough compute, and GPUs, which do not have enough communication, but is a good target for FPGAs.

Using the Izhikevich neuron model, each synaptic message can be represented by 48 bits [12]. Critical neuron parameters fill the FPGA BRAM, so space for packet buffers (for both message coalescing and retransmits) is very limited. With 128K neurons per FPGA, each FPGA generates 1.28M 48-bit synaptic messages per millisecond with a real-time deadline of arriving by the end of the next millisecond.

Worst-case throughput is therefore 1.28 billion messages per second from each FPGA. Due to spatial locality, some of these messages are for neurons that reside on the same FPGA and so can be stored in off-FPGA DRAM - the exact proportion depends on the neural network being simulated. The throughput requirements are therefore some percentage of the worst case.

\section{INTERCONNECT REQUIREMENTS}

In these application examples payload sizes are small (48 to 256 bits) and the application is latency-critical. Furthermore, the application does not have inbuilt support for retransmission: if a cache line request is dropped a CPU will simply stall, while a dropped neural message will introduce inaccuracy into a simulation.

When building our FPGA cluster, these applications led us to the following interconnect requirements:

1) Small message sizes: The interconnect must be able to efficiently deal with messages between 32 and 256 bits.

2) Low latency: Cluster applications are often more constrained by latency than bandwidth.

3) Reliable: With thousands of links each running at gigabits per second, errors are inevitable and could cause crashes or invalidate results.

4) Hardware-only: The interconnect must support reliable message delivery in hardware, without leaving reliability to software layers (as in TCP/IP).

5) Lightweight: The interconnect must use minimal FPGA area. This leaves more space for compute and permits use of smaller cheaper FPGAs.

6) Ubiquitous: The interconnect must maximize use of FPGA transceiver resources. More links and higher link rates means more bandwidth and fewer hops to cross a cluster.

7) Interoperable: The interconnect should be able to connect FPGAs of different types to build a heterogenous cluster.

\section{Bluelink: A Custom InTerconnect Toolkit}

To address the communication requirements of applications we created the BlueLink interconnect toolkit. An overview of BlueLink is shown in Figure 2. It has five major layers, written in Bluespec SystemVerilog:

1) Serial Transceiver: A hard core provided by an FPGA manufacturer. It is assumed that it implements $8 \mathrm{~b} 10 \mathrm{~b}$ coding and can be configured to send and receive 32-bit words with a 4-bit $k$ symbol indicator. BlueLink makes no assumptions 


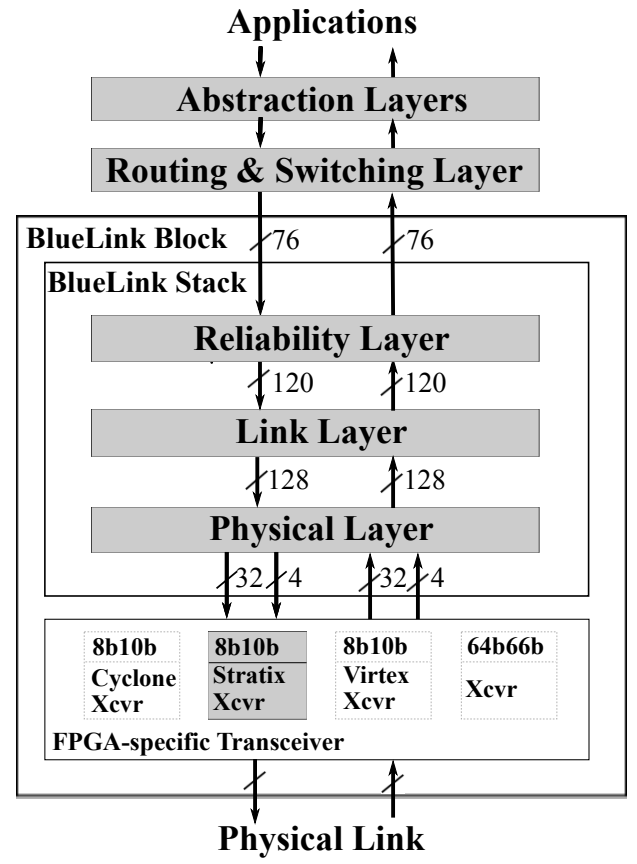

Fig. 2. Architecture of BlueLink interconnect

about the properties of a transceiver beyond its ability to successfully send and receive these $8 \mathrm{~b} 10 \mathrm{~b}$ symbols. Alternatively another coding scheme such as $64 \mathrm{~b} 66 \mathrm{~b}$ could be used. We have used Altera Stratix IV and Stratix V transceivers - it should be straightforward to use transceivers from other manufacturers.

BlueLink can use all the transceivers available on an FPGA board, over any physical medium. Currently SATA, PCIe, SMA, SFP+ copper and SFP+ optical cabling has been tested.

2) Physical: Transforms a FIFO-like stream of words from the Link layer into a continuous stream of words for the serial transceiver. Idle symbols and any alignment symbols required by the serial transceiver are inserted and removed as needed.

3) Link: Serializes 128-bit flits into 32-bit words on transmit and aligns these words back into flits on receive. Also performs clock crossing between the main FPGA clock domain and the transmit and receive clock domains of each transceiver.

4) Reliability: Implements reliable transmission with ordering and back-pressure.

5) Routing and switching: Uses a hop-by-hop routing scheme to direct packets to a given FPGA.

6) Application: Provides primitives for applications.

The Reliability and Application layers are described in further detail below.

The unit of reliable data transmission is a flit with a 64bit payload and 12-bit addressing field. This is expanded to 120 bits by the reliability layer by addition of a 32-bit CRC, a sequence number and an acknowledgement field. The physical layer adds a further 8-bit header, so that 128-bit flits are sent and received by the FPGA transceivers, often split into $4 \times 32$ bit words.

\section{A. Reliability layer}

The reliability layer is the first layer in the stack which does more than transforming and aligning symbols. It is tailored to meet the requirements identified in Section V.
It implements a reliable communication channel with FIFO semantics, providing a similar service to the TCP layer of the TCP/IP stack. However it is customized for small message sizes and low FPGA area. This means it must be economical with both header fields and memory buffers.

Reliability is implemented using a CRC and sequence number in each flit, which are validated by this layer in the receiving BlueLink block. A 32-bit CRC is used because the probability of false-negative is high in a large cluster with billions of flits per second. An acknowledgement number may also be appended to a flit to acknowledge correct receipt of a flit with that sequence number. If the receiver receives a flit which either fails the CRC or that is out of sequence, it does not send an acknowledgement. If there are pending acknowledgements to be sent but no input flits, a flit with no payload is sent for each acknowledgement.

Reliability is window-based, with transmitted flits that have not yet been acknowledged being stored in a replay buffer. If a flit is not acknowledged after a timeout (because the receiver detected an error or because an acknowledgement was lost), the flit at the head of the replay buffer is sent continuously until it is acknowledged, followed by every other flit in the buffer until the whole window of flits has been acknowledged. New flits are then accepted from the input. With 4-bit sequence and acknowledgement numbers the replay buffer only needs to hold $8 \times 64$-bit flits to store a whole retransmission window, a major contributor to the reduction in FPGA area requirements compared to other protocols that have longer flits/packets and larger windows.

Backpressure is achieved by sending acknowledgements with a flag to indicate that no more flits can be accepted. This prevents any further flits being transmitted, and so leads to the BlueLink block's input FIFO becoming full.

\section{B. Application abstractions}

A BlueLink block provides an Avalon Streaming interface to its clients. We have implemented a number of application abstractions to the BlueLink interconnect which match different communication paradigms and levels in the design hierarchy, to simplify partitioning applications over FPGA clusters.

1) Bluespec FIFO: Bluespec SystemVerilog is a dataflow hardware description language. Hardware modules are often connected using a FIFO abstraction rather than Verilog wires. This enables them to be easily decoupled while adding minimal logic overhead. BlueLink provides a Bluespec FIFO type that can be used to join two modules on different FPGAs. The only overhead is 10-20 extra cycles of latency compared with an on-chip FIFO.

2) Packets: BlueLink is also usable as a packet-based interconnect from software on custom processors. Hardware provides access to flit send and receive buffers. Traditional polling or interrupt mechanisms may be used to inform an application of packet delivery.

3) Blocking reads and writes: A lower-latency alternative to polling or interrupts is for a read or write to the flit buffer to block an application until it is performed successfully. This has lower overhead than polling as it is not necessary to spin in 
a loop until an operation can be performed. There is, however, a deadlock risk. Additionally it is possible to indicate a target FPGA by using part of the address of a write, which allows a flit to be sent in a single clock cycle.

A simple demonstration of this has been achieved by having a NIOS-II CPU executing code from DRAM located on another board. When the link cable is unplugged, the CPU pauses. When the cable is re-attached, the link resynchronizes and the CPU continues.

4) Remote DMA: A higher-level abstraction maps a wide range of memory addresses on each FPGA to a hardware module that performs remote DMA. Any read or write is translated to a read or write to a region of memory (or a memory-mapped peripheral) on a remote FPGA. A series of packets is sent to the hardware module on the remote FPGA, which performs the operation and returns the result as if it were a local operation.

Burst reads and writes are supported, enabling block transfers. Since it is not possible or desirable for an application to be aware of the details of the remote FPGA's memory map, such as the word size of a given memory device, bursts are translated into an appropriate sequence of operations at the remote device, including using byte enables with writes if a request does not align with word boundaries.

5) Software pipes: We also have an abstraction layer that emulates Linux pipe semantics. An application can be tested on a PC using Linux pipes between processes, then ported to the cluster and run unchanged.

These different abstractions provides a variety of primitives for partitioning applications. For example, the FIFO abstraction allows a hardware dataflow architecture to be split across FPGA boundaries, while the remote DMA abstraction means partitions can be viewed as nodes in a cluster-wide shared memory architecture.

\section{CORES FOR STANDARDIZED PROTOCOLS}

IP cores for standard communication protocols are commercially available from a number of vendors.

A natural assumption of someone building an FPGA cluster would be to use a popular protocol such as Ethernet. Ethernet is today a switched serial interconnect with data rates up to $100 \mathrm{Gbps}$. Interface cores, switches and cabling are commodity items. It is well understood, and is a convenient way to connect an FPGA cluster to a host PC. Some FPGA clusters such as the image retrieval accelerator in [10] are loosely coupled with no inter-FPGA communication. In this case Ethernet to a host PC may be a good fit for the application.

There are other protocols for which FPGA cores are available: Serial RapidIO, Infiniband, Interlaken, Fibre Channel, PCI Express and many more. We compare the characteristics of a selection of standard cores in Table I.

Notably the field can be divided into those protocols that have in-built support for reliability by packet retransmission, and those that do not. The performance of these varies widely, both in terms of physical link rate ${ }^{1}$ and area requirements.

\footnotetext{
${ }^{1}$ The computation of achievable bandwidth for diverse protocols is complex so we use link rate as a simple yardstick in this section. We provide more detailed case studies in our evaluation in Section VIII.
}

Ethernet has some restrictions for applications with tighter coupling. For example, [14] uses 37-bit payloads over Ethernet. To use the links efficiently these must be aggregated into packets, which results in latencies of $10 \mu$ s or more.

In addition Ethernet provides no native guarantee of packet delivery. In a cluster there may be thousands of links sending gigabits per second, so errors are inevitable and reliability is a necessity. TCP/IP is the conventional reliability mechanism, but is very expensive to handle in hardware [7]. For instance, clusters [10] and [14] did not consider it. For latency-sensitive applications, handling reliability in software is not an option. An alternative reliability protocol could be implemented on top of Ethernet - another example of custom communication.

PCI Express is commonly used for connecting FPGAs to a host PC. However it introduces a lot of complexity, being an emulation of traditional PCI over switched interconnect. For this reason FPGAs often have PCIe hard cores - but it is unusual for an FPGA to have more than one.

Interlaken is commonly used as a backplane interconnect in high-end switches. It is also very scalable, and relatively lightweight. There is also an optional retransmission extension. We tried to implement an Interlaken layer as an alternative to BlueLink, but came across the constraint that Altera's Stratix $\mathrm{V}$ core requires groups of eight or twelve bonded links to implement $50 \mathrm{G}$ or $100 \mathrm{G}$ channels. This was incompatible with the physical topology of the commodity Stratix V boards available to us. Altera also provide an alternative Interlaken core which requires groups of four or more channels, but it only works on the Stratix IV and has no reliability support.

Altera's SerialLite is an example of a lightweight vendorprovided protocol. SerialLite shares some similarities with BlueLink: SerialLite II provides packet retransmission of small packets. However it has been somewhat neglected - while it has been ported to modern FPGAs, its maximum link rate is 6 Gbps. Published area numbers are for Stratix II, and it is a commercial core for which a license is required. It is also incompatible with non-Altera FPGAs. We managed to synthesize a $6 \mathrm{G}$ SerialLite II core on a Stratix V, but the licensing restrictions did not allow us to test it on an FPGA.

SerialLite III is a modern version that runs at $10 \mathrm{Gbps}$ and beyond, however the protocol has been changed to support forward error correction preventing single bit errors. Across a cluster, where there may be thousands of links, cabling faults causing more substantial errors are likely so this protection is insufficient for our requirements. It is therefore only useful as a layer that does not guarantee correct packet transmission.

Aurora is Xilinx's equivalent to SerialLite, but has no reliability layer. This was used in systems such as an FPGA cluster [2] and a SoC prototyping system [9]. In both cases bit errors limited the usable link rate.

It became clear that using standard IP cores in an FPGA cluster can be fraught with practical difficulties:

1) Configuration constraints: Available parameters such as link rate and number of bonded lanes may not be appropriate.

2) Fitting requirements: A standard may require particular clock frequencies, PLLs or clock routing.

3) Bonded links: Useful on a custom PCB with skew-free parallel lanes between FPGAs. A commodity board and serial 
cabling may not have suitable configuration, either not enough lanes, unsuitable placement, or skew over different cables. Bonding can reduce the dimensions of the cluster compared with single links, adding hops and thus latency.

4) Manufacturer specific: Some protocols such as SerialLite and Aurora are only supported by one FPGA manufacturer. It is possible to implement these protocols on other FPGAs by reimplementing their specifications, but this would involve another core vendor or a custom implementation.

5) FPGA support: A core may only support some FPGA families, may be withdrawn in new tools, or not updated for new devices. It may require extensive reworking or prohibit using a newer FPGA.

6) Licensing: Designers must license IP cores from vendors, which can be expensive and can make evaluation difficult, particularly as a simulation of a link does not capture physical effects and so a license may be required for evaluation on a physical FPGA.

\section{Evaluation}

We evaluated BlueLink by synthesizing it on a Stratix V GX FPGA on a Terasic DE5-Net board and comparing with an implementation of Altera's existing 10G Ethernet MAC. The Stratix V platform was chosen to make a fair comparison between Ethernet and other existing standards that use 10G links - BlueLink is also capable of using lower-speed, lowercost FPGAs at $3 \mathrm{G}$ or $6 \mathrm{G}$ where Ethernet is often limited to $1 \mathrm{G}$. Ethernet does not provide reliable transmission while BlueLink does, so in practice another layer would be required above Ethernet. We attempted implementations of SerialLiteII and Interlaken but these were frustrated as described above.

Area comparison can be seen in Table II. 10G BlueLink uses $65 \%$ of the logic and registers of $10 \mathrm{G}$ Ethernet - indeed 40G BlueLink using bonded lanes will fit in about the same area as a single 10G Ethernet MAC. BlueLink also uses 15\% of the memory of 10G Ethernet. Compared with standard cores on Stratix V in Table I, BlueLink is more efficient than all the standards that support reliability and the majority that do not.

To consider throughput, we show the overhead of BlueLink and Ethernet-based packet structures in Figure 3. Our focus is on small packets, but BlueLink has higher throughput up to 256 bits. Using IP and/or TCP over Ethernet for reliability only serves to add additional overhead.

Figure 4 shows the latencies of BlueLink and Ethernet. We compare the latency of a link where the input queue is empty, and one where the link constantly receives input as fast as it can transmit. Both are tested on short physical links that have low error rates. Despite addition of a reliability layer with CRC checking, BlueLink's latency is about equivalent to Ethernet in the fully-loaded case. In the lightly-loaded case, BlueLink's latency is much lower as flits can be accepted in a single cycle, rather than the nine cycles that Altera's Ethernet core takes. As more transceivers are used on an FPGA it becomes more likely that links can be operated in this state where they are not fully loaded.

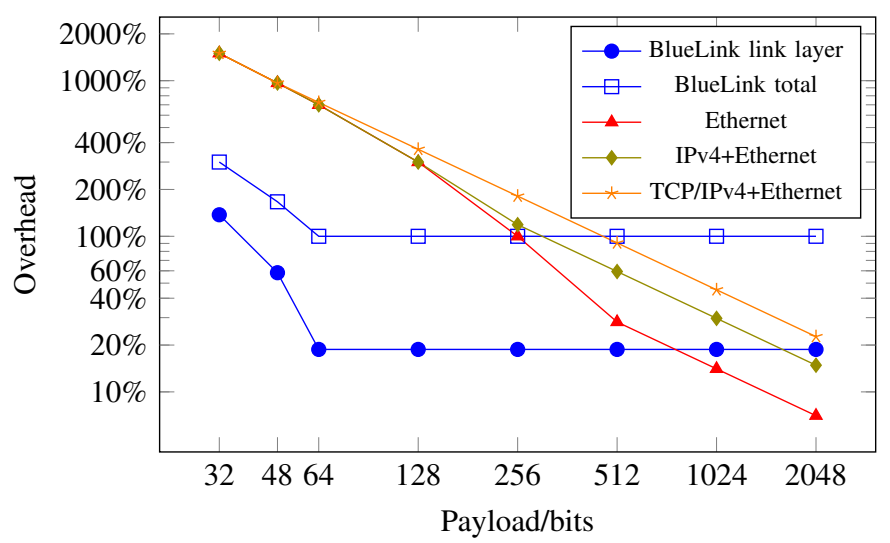

Fig. 3. Overhead of BlueLink against Ethernet-based standards for small packets. BlueLink makes considerably better use of bandwidth up to 256-bit packets.

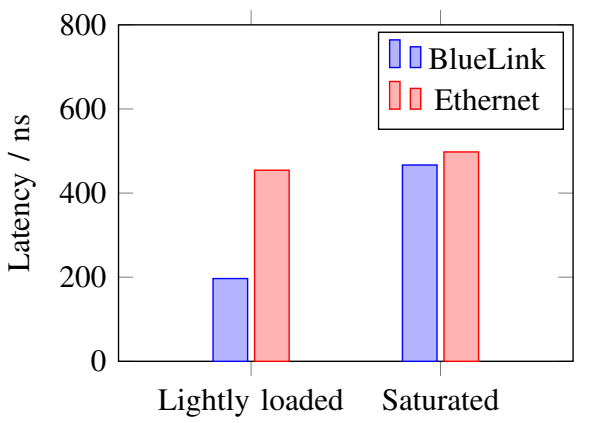

Fig. 4. Latency comparison between BlueLink and Ethernet MAC at 10 Gbps. BlueLink's latency compares favourably especially if many transceiver links can be used to keep the links lightly loaded.

Any FPGA system designer is faced with an area/performance tradeoff. This is particularly acute in modern FPGAs which have many transceivers. For comparison we take a Stratix V GX A7 FPGA, which is the lowest cost Stratix V that Terasic sell on an evaluation board. This FPGA has 48 transceivers each rated at $14.1 \mathrm{Gbps}$. We consider the situation that the designer wishes to use all the available transceivers. In Figure 5 we plot the FPGA area required for the different standards against the raw bandwidth provided. All standards are limited to $10 \mathrm{Gbps}$ per lane because this is the limit of commodity cabling (in theory BlueLink and SerialLite III will go higher). As can be seen, many standards have a considerable area penalty compared to a lightweight custom protocol such as BlueLink.

\section{A. Application example}

We used BlueLink as a key enabler for the Bluehive neural computation engine [12]. BlueLink was implemented on the DE4 Stratix IV 230 GX FPGA board from Terasic, which was chosen to maximise the number of DDR2 memory channels available. This is the middle of the Stratix IV range, much cheaper than high-end parts. To connect the boards we designed and open-sourced [17] a PCB to break out transceivers using PCI Express connectors into 6 Gbps SATA links (Figure 7). This enabled us to create a pluggable topology of low-cost SATA cables. Additional SATA cables were used directly in the FPGA boards' own SATA sockets. 


\begin{tabular}{|c|c|c|c|c|c|c|}
\hline System & $\begin{array}{l}\text { Raw external } \\
\text { link rate }\end{array}$ & Configuration & $\begin{array}{c}\text { Constituent } \\
\text { lane rate }\end{array}$ & LUTs & Registers & $\begin{array}{r}\text { Memory } \\
\text { bits }\end{array}$ \\
\hline \multicolumn{7}{|l|}{ Systems with reliable transmission } \\
\hline TCP/IP (in hardware) + Ethernet [7] & $10 \mathrm{G}$ & 1 lane & $10 \mathrm{G}$ & $<30000$ & Not quoted & Not quoted \\
\hline TCP datapath acceleration [6] (Virtex 6) & $10 \mathrm{G}$ & excluding CPU/MAC/PHY & & 6875 & 3889 & 221184 \\
\hline \multirow[t]{2}{*}{ SerialLite II (Stratix II 16 bit CRC) } & $6 \mathrm{G}$ & 1 lane & 6G & 1448 & 1236 & 90624 \\
\hline & 24G & 4 lanes & 6G & 2573 & 1659 & 176640 \\
\hline \multirow[t]{2}{*}{ PCIe hard IP } & $5 \mathrm{G}$ & 1x Gen 2 & $5 \mathrm{G}$ & 100 & 100 & 0 \\
\hline & $40 \mathrm{G}$ & $8 x$ Gen 2 & $5 \mathrm{G}$ & 200 & 200 & 0 \\
\hline \multirow{2}{*}{ PCIe soft IP (Stratix IV) } & $5 \mathrm{G}$ & 1x Gen 2 & $5 \mathrm{G}$ & 5500 & 4100 & 82944 \\
\hline & 20G & $4 x$ Gen 2 & $5 \mathrm{G}$ & 7100 & 5100 & 239616 \\
\hline \multirow[t]{2}{*}{ Serial RapidIO } & $5 \mathrm{G}$ & $1 \mathrm{x}$ & $5 \mathrm{G}$ & 5700 & 7885 & 737280 \\
\hline & $20 \mathrm{G}$ & $4 \mathrm{x}$ & $5 \mathrm{G}$ & 7200 & 10728 & 901120 \\
\hline Fibre Channel (Stratix IV) [13] & $8 \mathrm{G}$ & $1 \mathrm{x}$ & $8 \mathrm{G}$ & 3300 & 3900 & 6144 \\
\hline Infiniband [15] & $40 \mathrm{G}$ & LLC+TCA QDR 4x & $10 \mathrm{G}$ & 64105 & 63185 & 1584846 \\
\hline \multicolumn{7}{|c|}{ Systems that do not implement reliable transmission } \\
\hline Infiniband [15] & $40 \mathrm{G}$ & TCA QDR 4x & $10 \mathrm{G}$ & 36658 & 39912 & 1536807 \\
\hline SerialLite II (Stratix II) & $6 \mathrm{G}$ & $1 \mathrm{x}$ & $6 \mathrm{G}$ & 863 & 818 & 50688 \\
\hline SerialLite III ${ }^{\mathrm{ab}}$ & $120 \mathrm{G}$ & 12 lanes & $10.3125 \mathrm{G}$ & 5600 & 6200 & 983040 \\
\hline Aurora 8B/10B [16] & $12 \mathrm{G}$ & 4 lanes & $3 \mathrm{G}$ & 3473 & 3319 & 75776 \\
\hline Aurora 64B/66B [16] & $14 \mathrm{G}$ & 1 lane & $14 \mathrm{G}$ & 1600 & 1600 & 37920 \\
\hline Aurora 64B/66B [16] & $56 \mathrm{G}$ & 4 lanes & $14 \mathrm{G}$ & 3500 & 3900 & 43172 \\
\hline $1000 \mathrm{Mb}$ Ethernet MAC (external PHY) & $1 \mathrm{G}$ & 1 port RGMII & $125 \mathrm{Mx} 4 \mathrm{DDR}$ & 1184 & 1704 & 204976 \\
\hline 1000base-X Ethernet MAC & $1 \mathrm{G}$ & 1 lane & $1.25 \mathrm{G}$ & 1805 & 2365 & 204976 \\
\hline 10/100/1000Mb Ethernet MAC (ext. PHY) & $1 \mathrm{G}$ & 1 port RGMII & $125 \mathrm{Mx} 4 \mathrm{DDR}$ & 3155 & 3522 & 328064 \\
\hline 10/100/1000Mb Ethernet MAC (ext. PHY) & $1 \mathrm{Gx} 12$ & 12 port GMII & $125 \mathrm{Mx} 8 \mathrm{SDR}$ & 27360 & 29272 & 1479168 \\
\hline 10Gb Ethernet MAC & $10 \mathrm{G}$ & 1 lanes & $10.3125 \mathrm{G}$ & 2001 & 3077 & 0 \\
\hline 40Gb Ethernet MAC & 40G & 4 lanes & $10.3125 \mathrm{G}$ & 13600 & 23500 & 184320 \\
\hline 100Gb Ethernet MAC & $100 \mathrm{G}$ & 10 lanes & $10.3125 \mathrm{G}$ & 45100 & 87700 & 573440 \\
\hline Interlaken $100 \mathrm{G}^{\mathrm{a}}$ & $124 \mathrm{G}$ & 12 lanes & $10.3125 \mathrm{G}$ & 18900 & 36800 & 778240 \\
\hline Interlaken $50 \mathrm{G}^{\mathrm{a}}$ & $50 \mathrm{G}$ & 8 lanes & $6.25 \mathrm{G}$ & 12200 & 26300 & 942080 \\
\hline Interlaken 20G (Stratix IV) & $25 \mathrm{G}$ & 4 lanes & $6.25 \mathrm{G}$ & 12229 & 16774 & 479232 \\
\hline
\end{tabular}

${ }^{\mathrm{a}}$ Figures not available for optional reliability extension $\quad{ }^{\mathrm{b}}$ Provides insufficiently robust optional single bit error protection

TABLE I

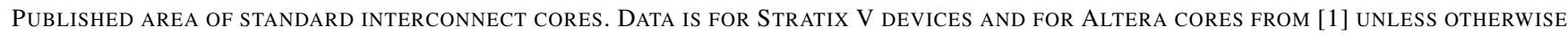
STATED. IN EACH CASE THE MINIMAL DESIGN HAS BEEN TAKEN - EXCLUDING PERFORMANCE COUNTERS AND OTHER OPTIONS.

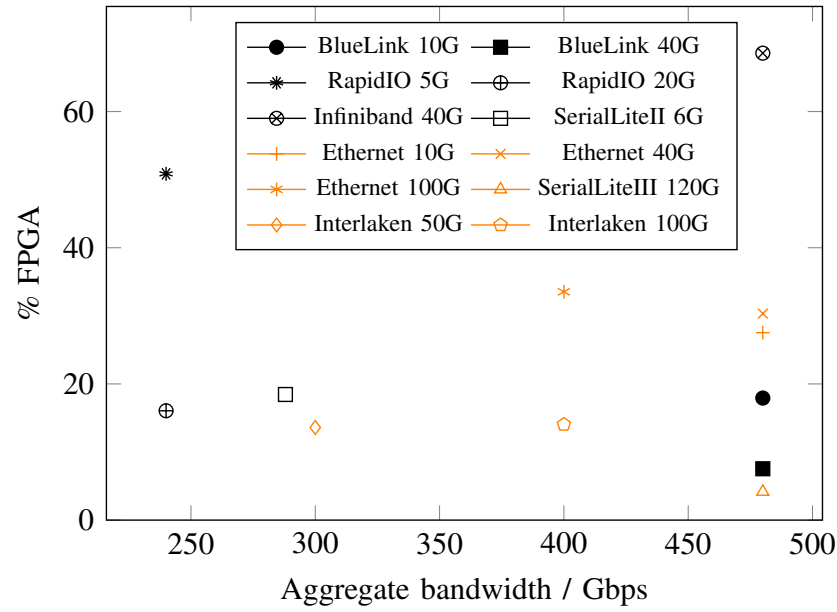

Fig. 5. Stratix V GX A7 logic utilization when instantiating each system as many times necessary to use all transceiver resources. Protocols in black implement reliability, those in orange do not, area numbers from Tables I and II. Multi-lane systems can share area between multiple transceivers and hence have lower area overall, but have additional fitting and routing constraints that make them more difficult to use in practice.

We put 16 DE4 boards together into a single Bluehive box (Figure 6), with the intention of the system scaling to further boxes using eSATA cables. We are currently working on building enclosures for 150 FPGAs.

To make a portable version we designed a PCB to join three FPGA boards using their PCIe $8 \times$ connectors (Figure 8 ) this is also able to connect Stratix V FPGAs with $40 \mathrm{Gbps}$ BlueLink bidirectional channels using groups of $4 \times 10 \mathrm{Gbps}$ lanes. Boards can also be joined with SFP+ cables.

Each FPGA hosts two custom soft vector processors, each

\begin{tabular}{lrrr}
\hline System & LUTs & Registers & Memory bits \\
\hline 10G BlueLink reliability layer & 1663 & 1277 & 2090 \\
10G BlueLink link layer & 179 & 413 & 960 \\
10G BlueLink PHY & 167 & 248 & 0 \\
\hline 10G BlueLink total area & $\mathbf{2 0 0 9}$ & $\mathbf{1 9 3 8}$ & $\mathbf{3 0 5 0}$ \\
\hline 40G BlueLink reliability layer & 1965 & 1355 & 2090 \\
40G BlueLink link layer & 1127 & 1970 & 2736 \\
40G BlueLink PHY & 289 & 585 & 0 \\
\hline 40G BlueLink total area & $\mathbf{3 3 8 1}$ & $\mathbf{3 9 1 0}$ & $\mathbf{4 8 2 6}$ \\
\hline 10G Ethernet MAC & 2986 & 3817 & 20972 \\
10G Ethernet PHY & 100 & 94 & 0 \\
\hline 10G Ethernet total area & $\mathbf{3 0 8 6}$ & $\mathbf{3 9 1 1}$ & $\mathbf{2 0 9 7 2}$ \\
\hline
\end{tabular}

TABLE II

AREA OF OUR IMPLEMENTATIONS OF BLUELINK AND ETHERNET ON A STRATIX V FPGA. BLUELINK USES LESS OF ALL RESOURCES, AND IN PARTICULAR MEMORY.

driving a DDR2-800 memory channel. These compute neural state updates and generate synaptic messages. The messages are then routed via BlueLink to other processors.

The system will successfully simulate two million neurons in near real-time. The application scales well - the limit on scaling is primarily compute bound, indicating that network bandwidth and latency have ceased to become a bottleneck.

\section{CONCLUSION}

The case for building an FPGA cluster from commodity evaluation boards with high-speed transceivers and commodity cabling is compelling. We have described how this approach solves a number of economic, physical and practical challenges faced by the system architect. It would therefore be a natural assumption that a commodity cluster should use commodity communication protocols. Our work has shown that this is not the case. 


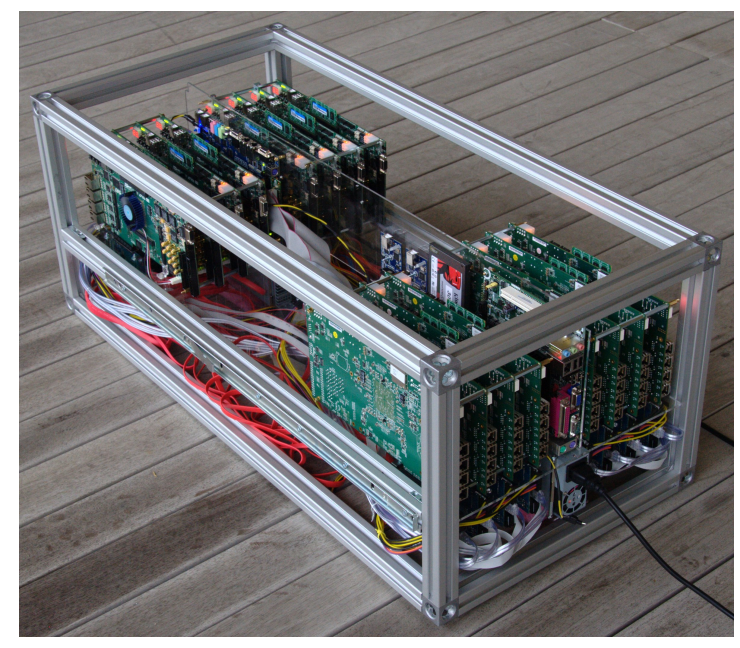

Fig. 6. Bluehive prototyping system

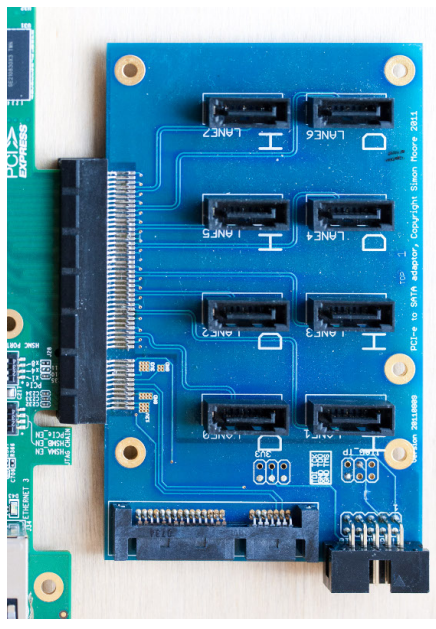

Fig. 7. PCIe to SATA breakout board

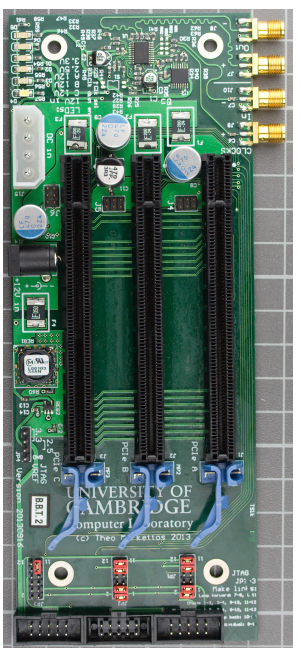

Fig. 8. PCB for BlueLink over PCIe connectors
Standard intellectual property (IP) cores are seductive. They give the promise of a 'drop-in' interconnect, a black box where the user need not be concerned with the internals. However, many FPGA to FPGA applications are different to those for which the protocols were designed. Library components bring with them a host of practical limitations that make their use more complex than might be expected.

We propose custom communication, by analogy with custom computation. A designer should consider their communication requirements at the same time as considering their compute requirements. An interconnect should then be designed from the ground up to meet the application's needs.

The interconnect must be lightweight and flexible to maximize use of FPGA transceivers, a resource which is growing rapidly in new FPGA families. The interconnect must also support reliable transmission of messages, because probabilities of error in a cluster are high and applications in hardware are not designed to handle packet error or loss.

Using the example of BlueLink, a custom interconnect toolkit we designed for a specific application, we have shown how FPGA application requirements can differ significantly from standard networking. Ethernet, which is a natural choice for networking, imposes significant overhead and latency penalties for the small messages used in our FPGA application. It also takes more area and lacks reliable transmission.

We have also evaluated a selection of other interconnect IP cores. Either they do not support reliability, leave little area for the application, have bandwidth limitations, or other restrictions. Resolving these problems can involve additional layers or wrappers to meet the application's requirements: an example of custom communication. A custom approach does not preclude the use of standard IP cores where they have useful properties; they may be components in a multi-layer stack. Design of such a stack should be considered from the beginning of the project. The designer should not simply reach for a standard IP core as the panacea for their needs.

\section{ACKNOWLEDGEMENTS}

This work was supported by the UK research council, EPSRC, grant EP/G015783/1. Additional funding was provided by the MRC2 Project that is sponsored by the Defense Advanced Research Projects Agency (DARPA) and the Air Force Research Laboratory (AFRL), under contract FA8750-11-C-0249. The views, opinions, and/or findings contained in this paper are those of the authors and should not be interpreted as representing the official views or policies, either expressed or implied, of the Defense Advanced Research Projects Agency or the Department of Defense.

\section{REFERENCES}

[1] Altera Corporation. Interface protocols. http://www.altera.co.uk/ products/ip/iup/ipm-index.jsp.

[2] T. Bunker and S. Swanson. Latency-optimized networks for clustering FPGAs. In Field-Programmable Custom Computing Machines (FCCM) 2013, pages 129-136.

[3] Altera Corporation. Stratix V GX FPGA development kit. http://www. altera.co.uk/products/devkits/altera/kit-sv-gx-host.html.

[4] DigiKey Corporation. Embedded - FPGAs. http: //www.digikey.com/product-search/en/integrated-circuits-ics/ embedded-fpgas-field-programmable-gate-array/2556262.

[5] DINI. Big FPGA boards for code verification and ASIC prototyping. http://www.dinigroup.com/new/why.php.

[6] DINI. TCP Offload Engine. http://www.dinigroup.com/new/TOE.php.

[7] Intilop Corporation. $10 \mathrm{G}$ bit TCP Offload Engine + MAC + Host_IF (SXTOE). http://www.intilop.com/ipcores.php.

[8] Y. Kono, K. Sano, and S. Yamamoto. Scalability analysis of tightlycoupled FPGA-cluster for lattice Boltzmann computation. In Field Programmable Logic and Applications (FPL) 2012, pages 120-127.

[9] Kouadri-Mostefaoui et al. Large scale on-chip networks : An accurate multi-FPGA emulation platform. In Digital System Design Architectures, Methods and Tools, 2008. 11th EUROMICRO Conference on, pages 3-9.

[10] Chen Liang et al. An FPGA-cluster-accelerated match engine for content-based image retrieval. In Field-Programmable Technology (FPT) 2013 , pages $422-425$.

[11] O. Mencer et al. Cube: A 512-FPGA cluster. In Southern Conference on Programmable Logic (SPL) 2009, pages 51-57.

[12] Simon W Moore, Paul J Fox, Steven J T Marsh, A Theodore Markettos, and Alan Mujumdar. Bluehive - a field-programable custom computing machine for extreme-scale real-time neural network simulation. In Proceedings of FCCM 2012, pages 133-140.

[13] MorethanIP. 1/2/4/8Gbps Fibre Channel Transport Core. http://www.morethanip.com/products_web/07_8gigabit_fibre/1_2_ 4_8_fc_fs_transport_pb_alt_v1.1.pdf.

[14] A.B. Nejad et al. An FPGA bridge preserving traffic quality of service for on-chip network-based systems. In Design, Automation Test in Europe Conference Exhibition (DATE), 2011, pages 1-6.

[15] Polybus Systems Corporation. InfiniBand cores. http://www.polybus. com/ib_link_layer_website/ib_cores_brochure_alt.pdf.

[16] ReFLEX CES. Focus on Aurora-like 8b/10b at 3Gbps. http://reflexces. com/focus.html?s=aurora-like-8b10b-3gbps.

[17] University of Cambridge. PCIe to SATA breakout board. http://www. cl.cam.ac.uk/research/comparch/opensource/pcie-sata/. 